\title{
Dieta Intermitente na Remodelação Cardíaca Induzida pelo Exercício físico
}

\author{
Intermittent Diet in Exercise-Induced Cardiac Remodeling
}

\author{
Francis Lopes Pacagnelli, ${ }^{1,2}$ Andreo Fernando Aguiar, ${ }^{3}$ Letícia Estevam Engel, ${ }^{1}$ Antônio Cláudio Bongiovani, ${ }^{2}$ \\ Mariana Janini Gomes ${ }^{4}$ \\ Programa de pós-graduação em Ciência Animal, Universidade do Oeste Paulista (Unoeste), ${ }^{1}$ Presidente Prudente, SP - Brasil \\ Programa de pós-graduação em Ciências da Saúde, Universidade do Oeste Paulista (Unoeste), ${ }^{2}$ Presidente Prudente, SP - Brasil \\ Programa de Pós-Graduação Profissional em Exercício Físico na Promoção da Saúde, Universidade Norte do Paraná (UNOPAR), ${ }^{3}$ Londrina, PR - Brasil \\ Divisão de Medicina Cardiovascular, Departamento de Medicina, Brigham and Women's Hospital, Harvard Medical School, ${ }^{4}$ Boston, MA - USA \\ Minieditorial referente ao artigo: Dieta Intermitente Atenua a Remodelação Cardíaca Causada pelo Exercício Físico
}

O artigo intitulado: 'Dieta Intermitente Atenua a Remodelação Cardíaca Causada pelo Exercício Físico' apresenta informações relevantes sobre os efeitos do exercício físico (EF) na remodelação cardíaca, capacidade funcional, comportamento nutricional e metabolismo glicêmico. Os autores também analisaram a expressão de proteínas associadas a diferenciação celular e remodelação cardíaca, tais como a extracellular signal-regulated kinase (ERK) e c-Jun $\mathrm{N}$-terminal kinase (JNK), a fim de determinar os possíveis mecanismos moleculares associados aos efeitos da dieta intermitente e EF. Os principais achados deste estudo foram que: 1) o EF isolado ou combinado a dieta intermitente aumentou a capacidade funcional, 2) a prescrição da dieta intermitente durante o programa de EF aumentou a tolerância glicêmica, 2) O EF isolado resultou na remodelação intersticial do miocárdio, e 3) a dieta intermitente foi capaz de atenuar a remodelação cardíaca induzida pelo $\mathrm{EF}^{1}{ }^{1}$

Vale ressaltar que este estudo utilizou um modelo experimental envolvendo ratos Wistar, a fim de simular uma dieta intermitente prescrita durante um programa de EF a longo prazo (12 semanas). ${ }^{1} \mathrm{~A}$ vantagem do modelo animal envolvendo aspectos nutricionais e físicos é a possibilidade de controlar as variáveis de confusão que podem afetar a validade interna da pesquisa, tais como o hábito alimentar, o estilo de vida, e a motivação intrínseca para o treino. Além disso, o modelo animal permite analisar os aspectos morfológicos, funcionais e moleculares do músculo cardíaco, possibilitando um estudo mais aprofundado dos mecanismos relacionados aos diversos tratamentos ou intervenções físicas e nutricionais. Desta forma, os resultados desse estudo são altamente consistentes e produzem informações relevantes para o entendimento e aprimoramento dos programas de prescrição de EF isolado ou associado a dieta intermitente. Esse estudo também aponta para uma nova direção de pesquisas sobre os efeitos do EF nos aspectos funcionais e morfológicos

\section{Palavras-chave}

Dieta Intermitente; Coração; Exercício.

Correspondência: Francis Lopes Pacagnelli •

Universidade do Oeste Paulista - Rua José Bongiovani. CEP 19050-920,

Presidente Prudente, Sp - Brasil

E-mail: francispacagnelli@unoeste.br

DOI: https://doi.org/10.36660/abc.20200632 da remodelação cardíaca, e como a dieta intermitente pode modular esses efeitos. ${ }^{1}$

Estudos com a finalidade de compreender os efeitos benéficos do EF isolado ou combinado a estratégias nutricionais são fundamentais para a prevenção e tratamento de doenças cardiovasculares. Tal assunto tem recebido especial atenção nos últimos anos, uma vez que a Organização Mundial da Saúde revelou um número de aproximadamente 17,2 milhões de mortes por doenças cardiovasculares a cada ano, o que representa sério problema de saúde pública, além dos elevados custos financeiros com tratamentos e internações hospitalares. ${ }^{2}$ Assim, o controle dos fatores de risco cardiovascular e a preocupação com hábitos de vida saudáveis são essenciais para prevenção e limitação de danos cardiovasculares, destacando a importância dos estudos envolvendo condutas não farmacológicas, tais como o EF e a nutrição.

Neste contexto, a dieta intermitente e o EF são intervenções não farmacológicas que vêm sendo preconizadas há décadas para a promoção da saúde e o tratamento de doenças cardiovasculares. Entre os diversos benefícios dessas estratégias estão melhora da qualidade de vida, da composição corporal, dos níveis de colesterol e triglicérides, da resistência à insulina, prevenção da hipertensão e do desenvolvimento de aterosclerose. ${ }^{3,4} \mathrm{O}$ impacto positivo da combinação de dieta intermitente e EF em diversas condições de saúde tem sido evidenciado em pesquisas experimentais e clínicas. ${ }^{5-7}$ Entretanto, a maioria dos estudos avaliaram os efeitos isolados do EF e da dieta intermitente, de modo que os efeitos da combinação destes tratamentos ainda não estão totalmente esclarecidos.

Estudo recente demonstrou o importante papel da dieta intermitente na redução de peso corporal e níveis de glicose sanguínea e de hemoglobina glicada, além de aumento da sensibilidade à insulina em camundongos obesos. ${ }^{8}$ Da mesma forma, o exercício aeróbio também tem demonstrado aumentar níveis proteicos musculares de GLUT 4 entre 20 a 70\% em humanos e roedores, contribuindo para melhorar a sensibilidade à insulina e melhor controle glicêmico. ${ }^{9}$ Logo, é provável que a combinação do EF com a dieta intermitente poderia ser uma estratégia benéfica para potencializar a melhora do metabolismo glicêmico. Esta hipótese é suportada por um recente estudo que reportou maior eficiência do EF aeróbio combinado a restrição calórica 
moderada, do que o EF isolado, na melhoria da aptidão cardiorrespiratória, fadiga, incapacidade e controle glicêmico em idosos obesos. ${ }^{10}$ Similarmente, no artigo de Basilio et al. ${ }^{1}$ os autores verificaram melhora da capacidade funcional e do metabolismo glicêmico em ratos submetidos ao EF isolado ou combinado a dieta intermitente. ${ }^{1}$ Tais achados reforçam a ideia de que o EF isolado ou combinado a restrição calórica pode ser benéfico para promoção da saúde global. ${ }^{1}$

Estudos com a finalidade de investigar os mecanismos celulares e moleculares envolvidos na remodelação cardíaca em resposta ao EF e a restrição calórica são fundamentais para o entendimento e aplicabilidade destas estratégias em programas de prevenção e reabilitação da função cardiovascular. O estudo de Basilio et al. ${ }^{1}$ avaliaram o papel de algumas proteínas quinases ativadas por mitógeno (MAPK) na remodelação cardíaca induzida pelo EF e na dieta intermitente. ${ }^{1}$ A proteína p38 MAPK, apesar de não ter sido avaliada nesse estudo, é uma das mais importantes proteínas da via das MAPK, pois é ativada em resposta a estímulos como o EF. Essa proteína pode modular a função dos fibroblastos cardíacos e o turnover na matriz extracelular e da indução parácrina da hipertrofia dos cardiomiócitos. ${ }^{11}$ Portanto, futuros estudos são necessários para avaliar o papel da p38 MAPK na remodelação cardíaca induzida pelo EF e se há influência com a dieta intermitente.

A remodelação do miocárdio é regulada por uma combinação de respostas dos cardiomiócitos e de outros tipos celulares a várias vias mecanosensíveis, as quais podem modificar a expressão gênica e síntese proteica e assim promover modificações funcionais nessas células. Estímulos mecanoreguladores como o exercício físico atuam nos cardiomiócitos e fibroblastos e levam a alterações na expressão gênica e remodelação celular. ${ }^{12}$ Pesquisas relatam a importância das integrinas, angiotensina II, cálcio e TGF $\beta$ regulando a via proteínas quinases ativadas do mitógeno (MAPK) com ativação dos fibroblastos e aumento da fibrose cardíaca ${ }^{12,13}$.

\section{Referências}

1. Basilio PG, Oliveira APC, Castro ACF, Carvalho MB, Zagatto AM, Martinez $\mathrm{PF}$, et al. Intermittent Fasting Attenuates Exercise Training-Induced Cardiac Remodeling. Arq Bras Cardiol. 2020; 115(2):184-193.

2. World Health Organization. (WHO). Available online: https://www.who. int/cardiovascular_diseases/en/ (accessed on 1 February 2019).

3. Malinowski B, Zalewska K, Węsierska A, Sokołowska MM, Socha M, Liczner G, et al. Intermittent fasting in cardiovascular disorders-an overview. Nutrients. 2019;11(3):673.

4. Gomes MJ, Pagan LU, Okoshi MP. Non-pharmacological treatment of cardiovascular disease | importance of physical exercise. Arq Bras Cardiol. 2019;113(1):9-10.

5. Wilson RA, Deasy W, Stathis CG, Hayes A, Cooke MB. Intermittent fasting with or without exercise prevents weight gain and improves lipids in dietinduced obese mice. Nutrients. 2018;10(3):346.

6. Moraes RCM, Portari GV, Ferraz ASM, da Silva TEO, Marocolo M. Effects of intermittent fasting and chronic swimming exercise on body composition and lipid metabolism. Appl Physiol Nutr Metab. 2017;42(12):1341-46.
Os efeitos da restrição calórica sobre a remodelação cardíaca têm sido muito investigados em modelos animais com alterações cardíacas. Foi demonstrado melhora da disfunção cardíaca e da reserva cronotrópica e, em relação a aspectos moleculares, melhora da inervação cardíaca simpática e níveis de receptores $\beta$-adrenérgicos em ratos com insuficiência cardíaca induzida por infarto do miocárdio submetidos a dieta intermitente. ${ }^{14}$ Outro estudo demonstrou que ciclos de jejum/realimentação promovem efeitos benéficos cardíacos e atenuam o dano miocárdico causado por restrição calórica em ratos espontaneamente hipertensos, contribuindo para reduzir o risco cardiovascular e os danos morfológicos. Além disso, o ciclo de jejum/ realimentação promove leve melhora do trânsito do $\mathrm{Ca}^{2+}$ e do sistema beta-adrenérgico. ${ }^{15} \mathrm{O}$ estudo de Basilio et al. não teve como objetivo investigar os efeitos do $\mathrm{EF}$ isolado ou combinado a restrição calórica sobre marcadores de trânsito do $\mathrm{Ca}^{2+}$, mas apresenta novas possibilidades de estudos sobre as adaptações moleculares relacionadas a remodelação cardíaca induzida pelo EF associado a dieta intermitente.. Ressaltamos a importância de testar os achados deste estudo em outras populações associadas a saúde e doenças, incluindo situações de sobrepeso, obesidade e diabetes, que cursam com alterações do metabolismo glicêmico.

Deste modo, vale novamente ressaltar que o artigo, ${ }^{1}$ apresenta valiosas informações sobre os efeitos do EF isolado ou combinado a dieta intermitente nos aspectos morfológicos e metabólicos envolvidos na remodelação cardíaca, contribuindo assim para o entendimento e aprimoramento dos programas de prevenção e reabilitação cardíaca.

\section{Agradecimentos}

Os autores agradecem Eric Schloeffel pela edição em inglês.
7. Bhutani S, Klempel MC, Kroeger CM, Trepanowski JF, Varady KA. Alternate day fasting and endurance exercise combine to reduce body weight and favorably alter plasma lipids in obese humans. Obesity (Silver Spring). 2013;21(7):1370-79

8. Gotthardt JD, Verpeut JL, Yeomans BL, Yang JA, Yasrebi A, Roepke TA, et al Intermittent fasting promotes fat loss with lean mass retention, increased hypothalamic norepinephrine content, and increased neuropeptide y gene expression in diet-induced obese male mice. Endocrinology. 2016;157(2):679-91.

9. Evans PL, McMillin SL, Weyrauch LA, \& Witczak CA. Regulation of skeleta muscle glucose transport and glucose metabolism by exercise training. Nutrients. 2019; 11(10):2432.

10. Nicklas BJ, Brinkley TE, Houston DK, et al. Effects of caloric restriction on cardiorespiratory fitness, fatigue, and disability responses to aerobic exercise in older adults with obesity: a randomized controlled trial. J Gerontol A Biol Sci Med Sci. 2019;74(7):1084-90.

11. Wang, Y. Mitogen-activated protein kinases in heart development and diseases. Circulation. 2007;116(12):1413-23. 


\section{Minieditorial}

12. Saucerman JJ, Tan PM, Buchholz KS, McCulloch AD, \& Omens JH. Mechanical regulation of gene expression in cardiac myocytes and fibroblasts. Nature Reviews Cardiology. 2019;16(6):361-78.

13. Molkentin, JD, Bugg D, Ghearing N, Dorn LE, Kim P, Sargent MA, et al. Fibroblast-specific genetic manipulation of p38 mitogen-activated protein kinase in vivo reveals its central regulatory role in fibrosis. Circulation. 2017;136(6):549-61.
14. de Lucia C, Gambino G, Petraglia L, Elia A, Komici K, Femminella GD, et al. Long-term caloric restriction improves cardiac function, remodeling, adrenergic responsiveness, and sympathetic innervation in a model of postischemic heart failure. Circulation: Heart Failure. 2018;11(3):e004153.

15. PinottiMF, Matias AM, Sugizaki MM, Nascimento AFD, Pai MD, Leopoldo AP, etal. Fasting/refeeding cycles prevent myocardial dysfunction and morphology damage in the spontaneously hypertensive rats. Arq Bras Cardiol. 2018; 111(3): 400-9. 